\title{
Strength of strong cycle connectivity in bipolar fuzzy graphs
}

\author{
S. Yahya Mohamed ${ }^{1 *}$ and N. Subashini ${ }^{2}$
}

\begin{abstract}
In this paper, the notion of cycle connectivity of bipolar fuzzy graphs is introduced. The concept of strong edge, $P$ cut node, $P$-bridge and $\theta$ - evaluation of bipolar fuzzy graphs are studied. The cycle connectivity of bipolar fuzzy trees, bipolar fuzzy cycles and complete bipolar fuzzy graphs are obtained. A condition for complete bipolar fuzzy graphs to have cyclic cut-nodes is obtained.

\section{Keywords}

$\theta$-evaluation, Bipolar Fuzzy Graph (BFG), Bipolar Fuzzy Strength (BFS), Bipolar Fuzzy Tree (BFT), $\alpha$-Strong, $\beta$-Strong and $\delta$-arc.

\section{AMS Subject Classification}

$05 \mathrm{C} 72$

${ }^{1}$ PG and Research Department of Mathematics, Government Arts College, Affiliated to Bharathidasan University, Tiruchirappalli-620022, Tamil Nadu, India.

${ }^{2}$ Research Scholar (Part-Time), Government Arts College, Affiliated to Bharathidasan University, Tiruchirappalli-620022, Tamil Nadu, India. *Corresponding author: ${ }^{*}$ yahya_md@yahoo.com; ${ }^{2}$ yazhinisuba@gmail.com

Article History: Received 24 October 2019; Accepted 28 December 2019

(C)2020 MJM
\end{abstract}

\section{Contents}

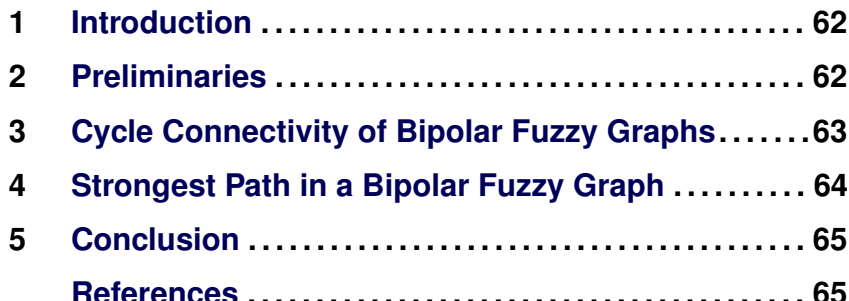

\section{Introduction}

A graph is a more suitable way of representing information involving relationship between objects. The objects are represented by vertices and relations by edges. The graph theoretical models are used to represent telephone network, railway network, traffic network, communication problems etc. In 1965, Zadeh [12] introduced the concept of a fuzzy subset of a set as a method for representing uncertainty. The theory of fuzzy sets has become a strong area of research in different disciplines including medical, management sciences, multi-agent systems, social sciences, computer networks engineering, life sciences statistics, graph theory, artificial intelligence, expert systems, signal processing, pattern recognition, robotics, decision making and automata theory.In 1994, Zhang
[13] introduced the concept of bipolar fuzzy sets as a generalization of fuzzy sets. Bipolar fuzzy sets are an extension of fuzzy sets whose membership degree range is $[-1,1]$. In 2011, Akram [2] introduced bipolar fuzzy graphs using bipolar fuzzy relation. Some recent works on fuzzy graphs can be referred in [1,3-11]. In this paper, the notion of cycle connectivity of bipolar fuzzy graphs is introduced. The concept of strong edge, $P$ - cut node, $P$-bridge and $\theta$ - evaluation of bipolar fuzzy graphs are studied. The cycle connectivity of bipolar fuzzy trees, bipolar fuzzy cycles and complete bipolar fuzzy graphs are obtained.

\section{Preliminaries}

Definition 2.1. A Bipolar Fuzzy Graph (BFG) is a pair $G=$ $(A, B)$ where $A=\left(\mu_{A}^{P}, \mu_{A}^{N}\right)$ is a bipolar fuzzy vertex in $V$ and $B=\left(\mu_{B}^{P}, \mu_{B}^{N}\right)$ is a bipolar fuzzy edge on $E$ such that $\mu_{B}^{P}(x, y)=\min \left(\mu_{A}^{P}(x), \mu_{A}^{P}(y)\right)$ and

$\mu_{B}^{N}(x, y)=\max \left(\mu_{A}^{N}(x), \mu_{A}^{N}(y)\right)$ for every $(x, y) \in E$.

Definition 2.2. $A B F G G=(A, B)$ is said to be a complete $B F G$ if $\mu_{B}^{P}(x, y)=\min \left(\mu_{A}^{P}(x), \mu_{A}^{P}(y)\right)$ and $\mu_{B}^{N}(x, y)=\max \left(\mu_{A}^{N}(x), \mu_{A}^{N}(y)\right)$ for all $x, y \in V$.

Definition 2.3. The positive strength of connectedness between two vertices $x$ and $y$ is defined as the maximum of 
the strength of all paths between $x$ and $y$ and is denoted by $\operatorname{CONN}_{G}^{P}(x, y)$. The negative strength of connectedness between two vertices $x$ and $y$ is defined as the minimum of the strengths of all paths between $x$ and $y$ and is denoted by $\operatorname{CONN}_{G}^{N}(x, y)$.

Definition 2.4. A $x-y$ path $P$ is called a strongest $x-y$ path if its positive and negative strength equals $\left(\operatorname{CONN}_{G}^{P}(x, y), \operatorname{CONN}_{G}^{N}(x, y)\right)$.

Definition 2.5. A weakest positive vertex of $G=(A, B)$ is a vertex with least membership value and weakest negative vertex of $G:(A, B)$ is a vertex with greatest membership value.

Definition 2.6. A path $P$ of length $n$ is a sequence of different vertices $\mu_{A}{ }^{P}\left(u_{0}\right), \mu_{A}{ }^{P}\left(u_{1}\right), \ldots, \mu_{A}{ }^{P}\left(u_{n}\right)$ and the degree of membership of a weakest edge is defined as its positive strength.

A path $P$ of length $n$ is a sequence of different vertices $\mu_{A}{ }^{N}\left(u_{0}\right), \mu_{A}{ }^{N}\left(u_{1}\right), \ldots, \mu_{A}^{N}\left(u_{n}\right)$ and the degree of membership of a greatest edge is defined as its negative strength.

Definition 2.7. An edge $(x, y)$ in BFG $G$ is called $\alpha$-strong if $\mu_{B}^{P}(x, y)>C O N N_{G-(x, y)}^{P}(x, y)$ and $\mu_{B}^{N}(x, y)<\operatorname{CONN}_{G-(x, y)}^{N}(x, y)$

Definition 2.8. An edge $(x, y)$ in BFG $G$ is called $\beta$-strong if $\mu_{B}^{P}(x, y)=C O N N_{G-(x, y)}^{P}(x, y)$ and $\mu_{B}^{N}(x, y)=C O N N_{G-(x, y)}^{N}(x, y)$.

Definition 2.9. An edge $(x, y)$ in $B F G G$ is called $\delta$-edge if $\mu_{B}^{P}(x, y)<C O N N_{G-(x, y)}^{P}(x, y)$ and $\mu_{B}^{N}(x, y)>C O N N_{G-(x, y)}^{N}(x, y)$.

Definition 2.10. An edge $(x, y)$ in BFG $G$ is called $\delta^{*}-$ edge if $\mu_{B}^{P}(x, y)>\mu_{B}^{P}(u, v)$ where $(u, v)$ is a weakest arc of $G$ and $\mu_{B}{ }^{N}(x, y)<\mu_{B}^{N}(u, v)$ where $(x, y)$ is weakest edge of $G$.

\section{Cycle Connectivity of Bipolar Fuzzy Graphs}

Definition 3.1. Let $G=(A, B)$ be a BFG. Then for any two vertices $\left(\mu_{A}^{P}(x), \mu_{A}^{N}(x)\right) \&\left(\mu_{A}^{P}(y), \mu_{A}^{N}(y)\right)$ of $G$, there is a set $\theta(x, y)$ called the $\theta$ - evaluation of $\left(\mu_{A}^{P}(x), \mu_{A}^{N}(x)\right), \quad \&\left(\mu_{A}^{P}(y), \mu_{A}^{N}(y)\right)$ and is defined as $\theta\left[\left(\mu_{A}^{P}(x), \mu_{A}^{N}(x)\right),\left(\mu_{A}^{P}(y), \mu_{A}^{N}(y)\right)\right]=$ $\{\alpha, \alpha \varepsilon[-1,1]\}$ where $\alpha$ is the strength of a strong cycle passing through both $\left(\mu_{A}^{P}(x), \mu_{A}^{N}(x)\right) \&\left(\mu_{A}^{P}(y), \mu_{A}^{N}(y)\right)$.

Note that if there is no strong cycle containing both $\left(\mu_{A}{ }^{P}(x), \mu_{A}{ }^{N}(x)\right) \&\left(\mu_{A}{ }^{P}(y), \mu_{A}^{N}(y)\right), \quad$ then define $\theta\left[\left(\mu_{A}^{P}(x), \mu_{A}^{N}(x)\right),\left(\mu_{A}^{P}(y), \mu_{A}^{N}(y)\right)\right]=\phi$.

\section{Definition 3.2.}

$$
\max \left\{\alpha \in \theta\left(\mu_{A}^{P}(u), \mu_{A}{ }^{N}(u)\right), \mu_{A}^{P}(v), \mu_{A}{ }^{N}(v) \varepsilon \sigma^{*}\right\}
$$

is defined as the cycle connectivity between $\left(\mu_{A}^{P}(u), \mu_{A}^{N}(u)\right) \&\left(\mu_{A}^{P}(v), \mu_{A}^{N}(v)\right) \quad$ in $\quad G:(\sigma, \mu)$ and is denoted by $C_{\left[\left(\mu_{A}{ }^{P}(u), \mu_{A}{ }^{N}(u)\right),\left(\mu_{A}{ }^{P}(v), \mu_{A}{ }^{N}(v)\right)\right]}$

$\theta\left[\left(\mu_{A}^{P}(u), \mu_{A}^{N}(u)\right),\left(\mu_{A}^{P}(v), \mu_{A}^{N}(v)\right)\right]=\emptyset$ for some pair of vertices $\left(\mu_{A}^{P}(u), \mu_{A}^{N}(u)\right) \&\left(\mu_{A}^{P}(v), \mu_{A}^{N}(v)\right)$ we define the cycle connectivity between $\left(\mu_{A}{ }^{P}(u), \mu_{A}^{N}(u)\right) \&\left(\mu_{A}^{P}(v), \mu_{A}^{N}(v)\right)$ to be $(0,0)$.

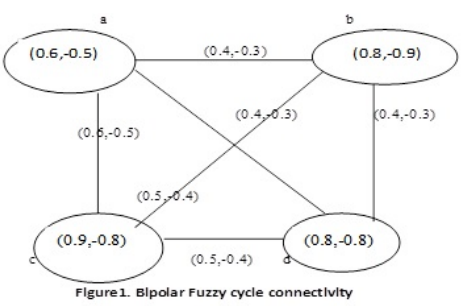

Figure 1. BFC

Example 3.3. Let $G:(A, B)$ be a BFG with $V=\{a, b, c, d\}$ and $E=\{a b, b c, a c, c d\}$. Then $\left[\mu_{B}^{P}(a b), \mu_{B}^{N}(a b)\right]=\left[\mu_{B}^{P}(b c), \mu_{B}^{N}(b c)\right]=\left[\mu_{B}^{P}(d b), \mu_{B}^{N}(d b)\right]=$ $(0.4,-0.3),\left[\mu_{B}^{P}(a c), \mu_{B}^{N}(a c)\right]=\left[\mu_{B}^{P}(d c), \mu_{B}^{N}(d c)\right]=$ $(0.5,-0.4)$ and $\left[\mu_{B}^{P}(a d), \mu_{B}^{N}(a d)\right]=(0.6,-0.5)$.

In Figure 1, bipolar fuzzy cycles are strong. Also there are only three bipolar fuzzy strong cycles passing through the vertex $\left[\mu_{A}^{P}(a), \mu_{A}^{N}(a)\right]$ and $\left[\mu_{A}^{P}(c), \mu_{A}^{N}(c)\right]$.

(i.e.,) Strength of $\{$ abca,adca,abcda $\}$ is $\{(0.4,-0.3),(0.5,-0.4),(0.4,-0.3)\}$ respectively.

Thus $\theta(a, c)=\{(0.4,-0.3),(0.5,-0.4)\}$ and hence $C_{a, c}^{G}=(0.5,-0.4)$

Definition 3.4. Let $G=(A, B)$ be a BFC connectivity of $G$ is defined as $\left[C C^{P}(G)=\max \left\{C_{u, v}^{P}(G) / u v \in \sigma^{*}\right\}\right.$ and $C C^{N}(G)=$ $\left.\min \left\{C_{u, v}^{N}(G) / u, v \in \sigma^{*}\right\}\right]$. That is cycle connectivity of a $B F G$ $G$ is defined as the maximum of positive cycle connectivity and minimum of negative cycle connectivity of distinct pair of vertices of $G$. Note in a BFG $G, C C(G)=(1,-1)$ if $G$ is a $B F C \& C C(G)=(0,0)$ if $G$ is a BFT.

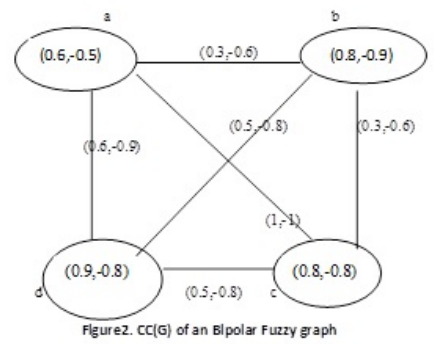

Figure 2. BFG

Example 3.5. Let $G=(A, B)$ be a $B F G$ with $V=\{a, b, c, d\}$ and $\left[\mu_{B}^{P}(a b), \mu_{B}^{N}(a b)\right]=\left[\mu_{B}^{P}(b c), \mu_{B}^{N}(b c)\right]=(0.3,-0.6)$ $\left[\mu_{B}^{P}(b d), \mu_{B}^{N}(b d)\right]=\left[\mu_{B}^{P}(d c), \mu_{B}^{N}(d c)\right]=(0.5,-0.8)$ and $\left[\mu_{B}^{P}(a c), \mu_{B}^{N}(a c)\right]=(1,-1),\left[\mu_{B}^{P}(a d), \mu_{B}^{N}(a d)\right]=(0.6,-0.9)$. Then $C_{(a, b)}^{G}=(0.3,-0.6), C_{(a, c)}^{G}=(0.5,-0.8)$, $C_{(a, d)}^{G}=(0.5,-0.8), C_{(b, c)}^{G}=(0.3,-0.6), C_{(b, d)}^{G}=(0.3,-0.6)$. 
Thus $C C(G)=(0.5,-0.8)$. Clearly the cycle connectivity of a $B F G$ is the maximum positive strength of a cycle and minimum negative strength of a cycle in $G$.

Theorem 3.6. $A$ BFGG is a BFT if and only if $C C(G)=$ $(0,0)$.

Proof. If $G$ is a BFG, then $C_{u, v}^{G}=(0,0)$ for each pair of vertices $\left(\mu_{A}{ }^{P}(u), \mu_{A}{ }^{N}(u)\right)$ and $\left(\mu_{A}{ }^{P}(v), \mu_{A}{ }^{N}(v)\right)$ in $G$. Here it follows that $C C(G)=(0,0)$.

Conversely suppose that $C C(G)=(0,0)$, Hence $C_{u, v}^{G}=$ $(0,0)$ for each pair of vertices in $G$. That is $G$ has no strong BFC. Hence $G$ has no BFC and hence G is a BFT.

Theorem 3.7. The cycle connectivity of a BFC is the strength of $G$.

Proof. The proof is obvious.

\section{Strongest Path in a Bipolar Fuzzy Graph}

Definition 4.1. Let $G$ be a BFG. A vertex $\left(\mu_{A}^{P}(w), \mu_{A}{ }^{N}(w)\right)$ is defined as $P$-cut vertex of $G$ if there exists a pair of vertices $\left(\mu_{A}{ }^{P}(u), \mu_{A}^{N}(u)\right),\left(\mu_{A}^{P}(v), \mu_{A}^{N}(v)\right)$ in $G$ such that $u \neq v \neq$ $w$ and

$\operatorname{CONN}_{G-(w)}^{P}(u, v)<\operatorname{CONN}_{G}^{P}(u, v) \&$

$\operatorname{CONN}_{G-(w)}^{N}(u, v)>\operatorname{CONN}_{G}^{N}(u, v)$.

A connected BFG having no P-cut vertices is called a P-block.

Example 4.2. Let $G:(V, E)$ be BFG given in Fig.3

Note that $b$ is a $P$-cut vertex since $\operatorname{CONN}_{G-b}^{P}(a, c)=0.5<$ $0.9=\operatorname{CONN}_{G}^{P}(a, c) \& C O N N_{G-b}^{N}(a, c)=-0.4>-0.8=$ $\operatorname{CONN}_{G}^{N}(a, c)$.

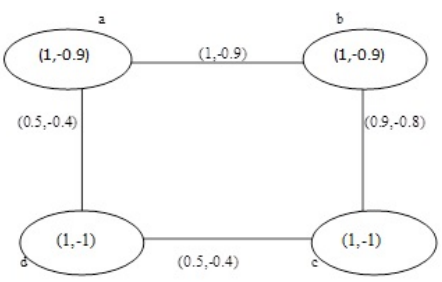

Figure 3. BFG

Definition 4.3. Let $G=(A, B)$ be a BFG. An edge $e=\left(\mu_{B}^{P}(u, v), \mu_{B}^{N}(u, v)\right) \in B$ is called a $P$ bridge if $\operatorname{CONN}_{G-e}^{P}(u, v)<\operatorname{CONN}_{G}^{P}(u, v)$ and $\operatorname{CONN}_{G-e}^{N}(u, v)>\operatorname{CONN}_{G}^{N}(u, v) . \quad$ A P-bridge is called P-bond if $\operatorname{CONN}_{G-e}^{P}(x, y)<\operatorname{CONN}_{G}^{P}(x, y)$ and $\operatorname{CONN}_{G-e}^{N}(x, y)>\operatorname{CONN}_{G}^{N}(x, y)$ with atleast one of $x$ or $y$ different from both $u$ and $v$, and is said to be a P-cut bond if both $x$ and $y$ are different from $u$ and $v$.

Example 4.4. Consider the following BFG with four vertices. Here all edges except edge $(a, d)$ are $P$ - bonds. In particular edge $(b, c)$ is a $P$-cut bond. Since $\operatorname{CONN}_{G-(b, c)}^{P}(a, d)=0.5<$

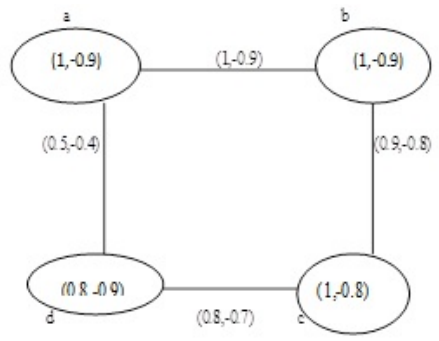

Figure 4. BFG with p-cut bond

$0.8=\operatorname{CONN}_{G}^{P}(a, d)$ and $\operatorname{CONN}_{G-(b, c)}^{N}(a, d)=-0.4>$ $-0.7=\operatorname{CONN}_{G}^{N}(a, d)$.

Theorem 4.5. In a BFC all edges are strong edges.

Proof. Let $G$ be a BFC. Let all edgesare not strong edges. Then there exist atleast one weak edge in G. Let us discuss the proof in two cases.

Case i:

Only one weak edge exists. The positive membership value of the edge is minimum and the negative membership value of the edge is maximum. But in bipolar fuzzy cycle there should be atleast two positive edges with minimum membership value and atleast two negative edges with maximum membership value which is a contradiction to our assumption. Case ii:

More than two positive edges with minimum membership value and more than two negative edges with maximum membership value. Then the edges will not be weak edges.

Theorem 4.6. A connected BFG $G$ is a P-block if and only if any two vertices $\left(\mu_{A}^{P}(u), \mu_{A}^{N}(u)\right),\left(\mu_{A}^{P}(v), \mu_{A}^{N}(v)\right) \in$ $V(G)$, such that $(u, v)$ is not $\alpha$ - strong are joined by two internally disjoint strongest paths.

Proof. Assume that G is a P-block. Let $\left(\mu_{A}^{P}(u), \mu_{A}^{N}(u)\right)$, $\left(\mu_{A}{ }^{P}(v), \mu_{A}{ }^{N}(v)\right) \in V(G)$, such that (u,v) is not $\alpha$ - strong. To prove that there exist two internally disjoint strongest u-v paths. Suppose not. There exists exactly one internally disjoint strongest $\mathrm{u}-\mathrm{v}$ path in G. Since $(\mathrm{u}, \mathrm{v})$ is not $\alpha-$ strong, length of all strongest $\mathrm{u}-\mathrm{v}$ paths must be atleast two. Also for all strongest $\mathrm{u}-\mathrm{v}$ paths in $\mathrm{G}$, there must a vertex in common. Let $\left(\mu_{A}^{P}(w), \mu_{A}^{N}(w)\right)$ be such a vertex in G. Then $\operatorname{CONN}_{G-w}^{P}(u, v)<\operatorname{CONN}_{G}^{P}(u, v) \& \operatorname{CONN}_{G-w}^{N}(u, v)>$ $\operatorname{CONN}_{G}^{N}(u, v)$ which contradicts the fact that $\mathrm{G}$ has no P-cut vertex. Conversely suppose that any two vertices of $\mathrm{G}$ are joined by two internally disjoint strongest paths. Let $\left(\mu_{A}{ }^{P}(w), \mu_{A}^{N}(w)\right)$ be a vertex in G. For any pair of verticesu,v $\in V(G)$ such that $\left(\mu_{A}^{P}(u), \mu_{A}^{N}(u)\right) ?\left(\mu_{A}^{P}(v), \mu_{A}^{N}(v)\right) ?\left(\mu_{A}^{P}(w), \mu_{A}^{N}(w)\right)$. There always exists a strongest path not containing $\left(\mu_{A}{ }^{P}(w), \mu_{A}^{N}(w)\right) . \quad$ So $\quad\left(\mu_{A}{ }^{P}(w), \mu_{A}^{N}(w)\right)$ cannot be a p-cut vertex and the theorem is proved. 
Theorem 4.7. Let $G$ be a connected BFG and let $\left(\mu_{A}^{P}(x), \mu_{A}^{N}(x)\right) \&\left(\mu_{A}^{P}(y), \mu_{A}^{N}(y)\right)$ be any two vertices in $G$. Then there exists a strong path from $x$ to $y$.

Proof. Assume that $\mathrm{G}$ is a connected BFG and let $\left(\mu_{A}{ }^{P}(x), \mu_{A}^{N}(x)\right) \&\left(\mu_{A}{ }^{P}(y), \mu_{A}^{N}(y)\right)$ be any two vertices of $\mathrm{G}$. If an edge $(\mathrm{x}, \mathrm{y})$ is strong, there is immediate result. Otherwise, either $(\mathrm{x}, \mathrm{y})$ is a $\delta$-edge or there exist a path of length more than one from $\mathrm{x}$ to $\mathrm{y}$. In the first case we can find a path $\mathrm{P}$ (say) such that $S^{P}(P)>W^{P}(x, y) \& S^{N}(P)<W^{N}(x, y)$. In either case consider the path from $\mathrm{x}$ to $\mathrm{y}$ of length more than one. If some edge on this path is not strong, replace it by a path having more strength. This argument cannot be repeated arbitrary often: hence eventually we can find a path from $x$ to $\mathrm{y}$ on which all the edges are strong.

Theorem 4.8. An edge $(u, v)$ in a $B F G G=(A, B)$ is a strongest $u-v$ path if and only if $(u, v)$ is either $\alpha$-strong or $\beta$-strong.

Proof. Let $G=(A, B)$ be a BFG. Let $(u, v)$ be an edge in $\mathrm{G}$. Consider the path $\mathrm{P}: u-v$ in $\mathrm{G}$. Then by definition of strength of a path, Strength of $\mathrm{P}=\left(\mu_{B}{ }^{P}(u, v), \mu_{B}{ }^{N}(u, v)\right)-(1)$ Let $\mathrm{P}$ is a strongest path, then strength of $\mathrm{P}=\left(\operatorname{CONN}_{G}^{P}(u, v), \operatorname{CONN}_{G}^{N}(u, v)\right) \quad$ From 1, $\left(\mu_{B}^{P}(u, v), \mu_{B}^{N}(u, v)\right)=\left(\operatorname{CONN}_{G}{ }^{P}(u, v), \operatorname{CONN}_{G}{ }^{N}(u, v)\right)$ Strength of positive path $(\mathrm{P}) \geq$ Strength of all other $\mathrm{u}-\mathrm{V}$ paths \& Strength of negative path $(\mathrm{P}) \leq$ Strength of all other $\mathrm{u}-\mathrm{v}$ paths In particular, Strength of positive path $(\mathrm{P}) \geq \operatorname{CONN}_{G-(u, v)}^{P}(u, v) \&$ Strength of negative path $(\mathrm{P}) \leq \operatorname{CONN}_{G-(u, v)}^{N}(u, v)$ Thus $\operatorname{CONN}_{G}^{P}(u, v) \geq \operatorname{CONN}_{G-(u, v)}^{P}(u, v)$ and $\operatorname{CONN}_{G}^{N}(u, v) \leq \operatorname{CONN}_{G-(u, v)}^{N}(u, v)-$-(3)

Now from (2) and (3), $\mu_{B}^{P}(u, v) \geq \operatorname{CONN}_{G-(u, v)}^{P}(u, v)$ and $\mu_{B}^{N}(u, v) \leq \operatorname{CONN}_{G-(u, v)}^{N}(u, v)$, edge $(\mathrm{u}, \mathrm{v})$ is either $\alpha$-strong or $\beta$-strong. Conversely assume that edge $(\mathrm{u}, \mathrm{v})$ is either $\alpha$-strong or $\beta$-strong. Then $\mu_{B}^{P}(u, v) \geq$ $\operatorname{CONN}_{G-(u, v)}^{P}(u, v) \& \mu_{B}^{N}(u, v) \leq \operatorname{CONN}_{G-(u, v)}^{N}(u, v)$

$$
\left[\operatorname{CONN}_{G}^{P}(u, v), \operatorname{CONN}_{G}^{N}(u, v)\right]=\left[\mu_{B}^{P}(u, v), \mu_{B}^{N}(u, v)\right]
$$

i.e., $\left[\operatorname{CONN}_{G}^{P}(u, v), \operatorname{CONN}_{G}^{N}(u, v)\right]=$ Strength of $P$. Therefore $P$ is a strongest path in $G$.

Theorem 4.9. Let $G=(A, B)$ be a $B F G$ and let $(u, v)$ be any edge in a $u_{0}-u_{n}$ path $P$ such that Strength of $P=$ $\left[\mu_{B}^{P}(u, v), \mu_{B}^{N}(u, v)\right]$. Then $P$ is a strongest $u_{0}-u_{n}$ path if $(u, v)$ is a strong edge and it is the unique weakest edge of $P$.

Proof. Let $G=(A, B)$ be a BFG. Let P: $u_{0}-u_{1}-$ $u_{2} \cdots-u_{n}$ be $u_{0}-u_{n}$ path in $\mathrm{G}$ where Strength of $\mathrm{P}=$ $\left[\mu_{B}^{P}\left(u_{i-1}, u_{i}\right), \mu_{B}^{N}\left(u_{i-1}, u_{i}\right)\right]$ for some $\mathrm{i}=1,2,3 \ldots \mathrm{n}$. Let $\left(u_{i-1}, u_{i}\right)$ be a strong edge and let it be the unique weakest edge in $\mathrm{P}$. To show that $\mathrm{P}$ is a strongest $u_{0}-u_{n}$ path. If possible suppose $\mathrm{p}$ is not a strongest $u_{0}-u_{n}$ path. Let : $P_{1}: u_{0}-v_{1}-$ $v_{2} \ldots \ldots v_{n-1}-u_{n}$ be a strongest $u_{0}-u_{n}$ path in $\mathrm{G}$, where all of $u_{i}, i=1,2,3, \ldots n-1$ and $v_{j}, j=1,2,3, \ldots n-1$ need not be different. Since strength of $P_{1}$ is greater than strength of $\mathrm{P}$, we have strength of every positive edge of $P_{1}>\mu_{B}^{P}\left(u_{i-1}, u_{i}\right)$ and strength of each negative edge of $P_{1}<\mu_{B}^{N}\left(u_{i-1}, u_{i}\right)$. Also note that edge $\left(u_{i-1}, u_{i}\right)$ is not a common edge for $P$ and $P_{1}$. Then $P \cup P_{1}$ will contain atleast one cycle and let $C$ be one such cycle in which the only weakest edge is $\left(u_{i-1}, u_{i}\right)$. Consider a $\left(u_{i-1}, u_{i}\right)$ path $P^{\prime}$ in $C$ not containing the edge $\left(u_{i-1}, u_{i}\right)$. Clearly $\mu_{B}^{P}\left(u_{i-1}, u_{i}\right)<$ Strength of positive path $P^{\prime}$ and $\mu_{B}^{N}\left(u_{i-1}, u_{i}\right)>$ Strength of negative path $P^{\prime}$ and strength of positive path $P^{\prime}=\operatorname{CONN}_{G-\left(u_{i-1}, u_{i}\right)}^{P}\left(u_{i-1}, u_{i}\right)$ and strength of negative path $P^{\prime}=\operatorname{CONN}_{G-\left(u_{i-1}, u_{i}\right)}^{N}\left(u_{i-1}, u_{i}\right)$ $\therefore \mu_{B}^{P}\left(u_{i-1,}, u_{i}\right)<\operatorname{CONN}_{G-\left(u_{i-1}, u_{i}\right)}^{P}\left(u_{i-1}, u_{i}\right)$ and $\mu_{B}^{N}\left(u_{i-1}, u_{i}\right)>\operatorname{CONN}_{G-\left(u_{i-1}, u_{i}\right)}^{N}\left(u_{i-1}, u_{i}\right)$ which implies $\left(u_{i-1}, u_{i}\right)$ is a $\delta$ - edge, which contradicts that $\left(u_{i-1}, u_{i}\right)$ is a strong edge.

Hence $\mathrm{P}$ must be a strongest $u_{0}-u_{n}$ path in $\mathrm{G}$.

\section{Conclusion}

BFGs are the precise models of all kinds of networks. As the reduction in the strength of connectedness is more important than the total disconnection of the network, this new concept can be applied to study the connectivity parameters of networks. This will be carried out in the forthcoming papers.

\section{Acknowledgment}

The authors are grateful to the referees for these valuable and constructive suggestions.

\section{References}

[1] M. B. Ahamed, Types of degrees in bipolar fuzzy graphs, Appl. Math. Sci. (Ruse), 7(2013), 4857-4866.

[2] M.Akram, Bipolar fuzzy graphs, Information Sciences, 2011, 12-23.

[3] M. Akram, W. A. Dudek, Regular bipolar fuzzy graphs, Neural Comput. Appl., 21(2012), 197-205.

[4] M. Akram, N. Waseem, Novel applications of bipolar fuzzy graphs to decision making problems, J. Appl. Math. Comput., 56(2018), 73-91.

[5] S. Mathew and M.S. Sunitha, Cycle connectivity in fuzzy graphs, Journal of Intelligent and Fuzzy System, 24(2013), 549-554.

[6] S. Yahya Mohamed, A. Mohamed Ali , Interval-Valued Pythagorean Fuzzy Graph, Journal of Computer and Mathematical Sciences, 9(10)(2018), 1497-1511.

[7] S. Yahya Mohamed, A. Mohamed Ali, Modular product on intuitionistic fuzzy graphs, International Journal of Innovative Research in Science, Engineering and Technology, 6(9)(2017), 19258-19263.

[8] S.Yahya Mohamed and N. Subashini, Connectivity in a Bipolar Fuzzy Graph and its Complement, Annals of Pure and Applied Mathematic, 15(1)(2017), 89-95. 
[9] S.Yahya Mohamed and N. Subashini, Structures of edge regular bipolar fuzzy graphs, International Journal of Mathematics Trends and Technology, 58(3)(2018), 215220.

[10] S. Yahya Mohamed and N. Subashini, Bipolar Fuzzy Graphs Based on Eccentricity Nodes, Journal of Applied Science and Computations, 5(11)(2018), 153-158.

[11] S. Yahya Mohamed and N. Subashini, Some types of paths in a Bipolar Fuzzy Graphs, Journal of Applied Science and Computations, 6(6)(2019), 305-308.

${ }^{[12]}$ L.A.Zadeh, Fuzzy sets, Information and control, 8(1965), 338-353.

[13] W. R. Zhang, Bipolar fuzzy sets and relations: a computational framework for cognitive modeling and multi-agent decision analysis, Proceedings of the First International Joint Conference of The North American Fuzzy Information Processing Society Biannual Conference, 1994 (1994), 305-309.

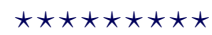

$\operatorname{ISSN}(\mathrm{P}): 2319-3786$

Malaya Journal of Matematik

$\operatorname{ISSN}(\mathrm{O}): 2321-5666$

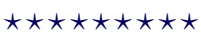

